\title{
Search for 'exotic' baryons at HeRmes
}

\author{
Wouter Deconinck \\ for the HERMEs Collaboration
}

UNIVERSITY OF MICHIGAN

Quarks and Nuclear Physics 2006

June 5, 2006 


\section{Overview}

- Motivation

- $\Theta(1540)$ at HeRmes

- Photo-production of $\Lambda(1520)$ and $\Theta(1540)$

- Hadron photo-production

- The Hermes Spectrometer

- Event selection and reconstruction

- $\Lambda(1520)$ spectra and cross section

- Conclusions 


\section{Motivation}

\section{Exotics at HERMES}

- $\Theta(1540)$ observed (59 \pm 16 events) $M=1528 \pm 2.6$ (stat) \pm 2.1 (syst) $\mathrm{MeV}$

- $\bar{\Theta}(1540)$ not observed ( $3 \pm 6$ events)
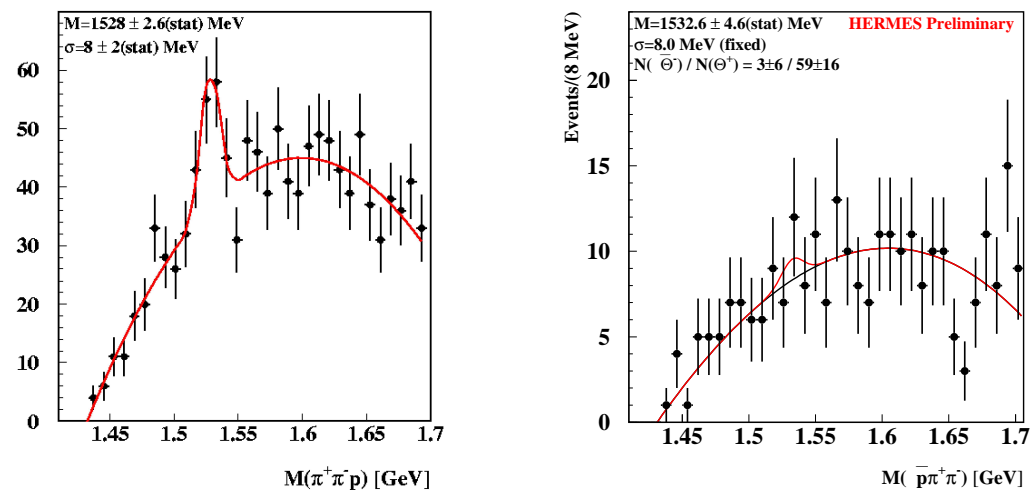


\section{Motivation}

Photo-production of $\Lambda(1520)$ and $\Theta(1540)$

- In some models: production mechanism similar

- Assumption: anti-particle/particle ratio similar

- Determine cross section ratios $\bar{\Lambda} / \Lambda, \Theta / \Lambda, \bar{\Theta} / \bar{\Lambda}$
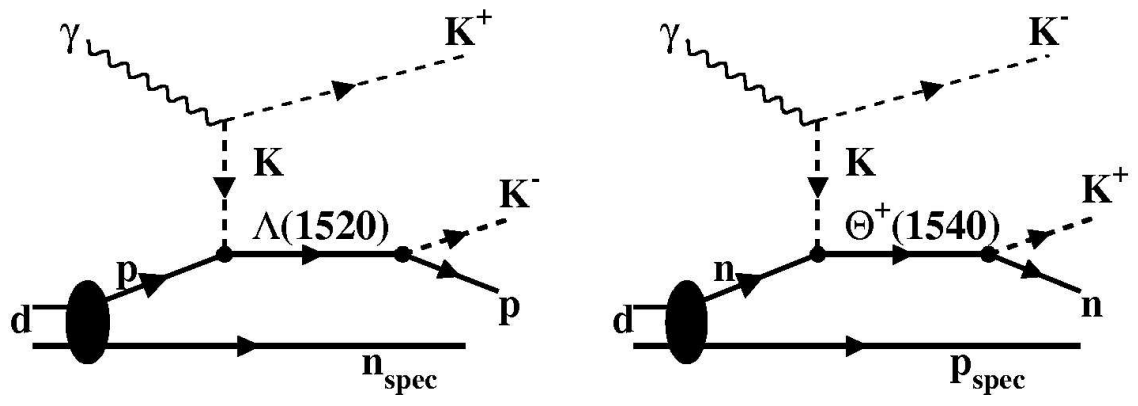


\section{Motivation}

\section{Hadron production}

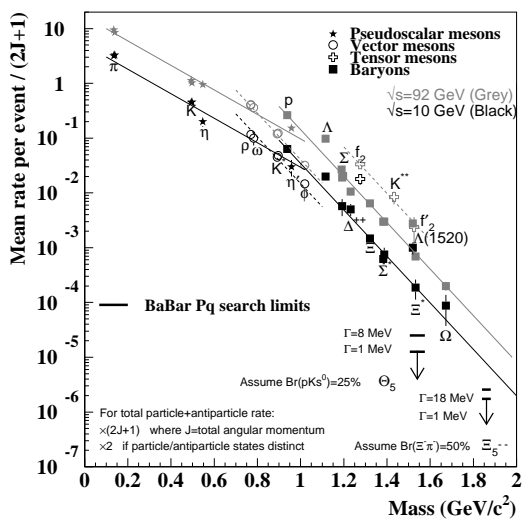

BaBar: search for $\Theta^{+}$and $\Xi^{--}$
Mesons and baryons

- BaBar: ee $\rightarrow(q \bar{q})^{n} \rightarrow X$

- Different slope for mesons and baryons!

\section{Exotics at HERMES}

- HeRMes: photo-production

- Do exotics have a different slope?

- Where is our $\sigma_{\Theta}$ ? 


\section{The HERMES Spectrometer}

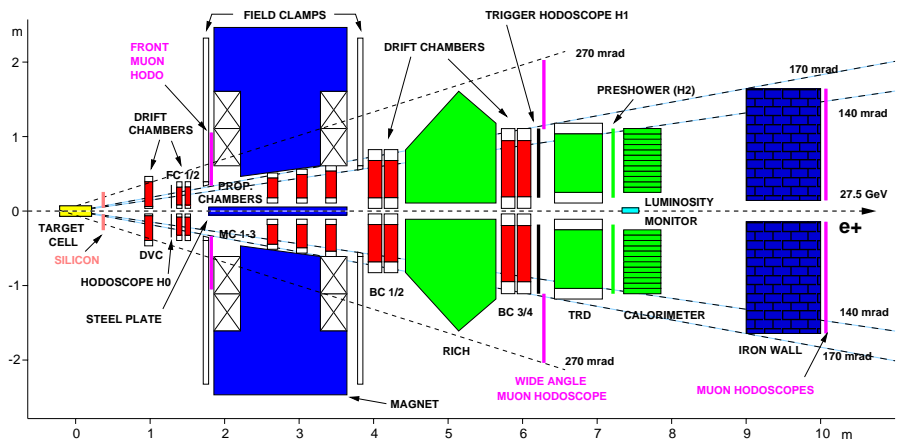

- 27.6 GeV $e^{ \pm}$HERA beam on $\vec{H}, \overrightarrow{H e}, \vec{D}$ or $\mathrm{H}_{2}, \mathrm{D}_{2}, \mathrm{He}, \ldots$

- Resolution: $\frac{\Delta p}{p}=1.4-2.5 \%, \Delta \vartheta \lesssim 0.6 \mathrm{mrad}$

- TRD, Calorimeter and Preshower: hadron/lepton separation

- RICH: hadron identification $(p, \pi, K)$ 


\section{The HERMES Spectrometer}

Hadron/lepton separation: combination of

- TRD

- Calorimeter

- Preshower

- $\mathrm{RICH}$

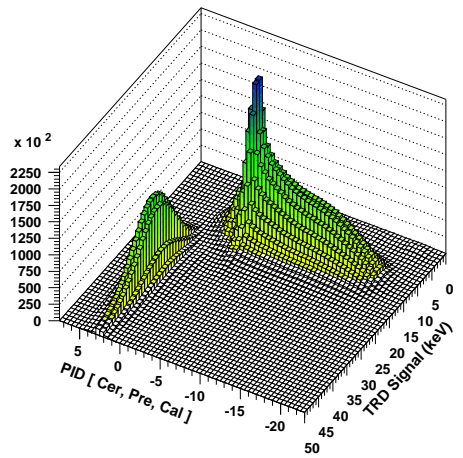

Hadron identification: dual radiator $\mathrm{RICH}$

- aerogel: $n=1.03$

- $\mathrm{C}_{4} \mathrm{~F}_{10}$ gas: $n=1.0014$

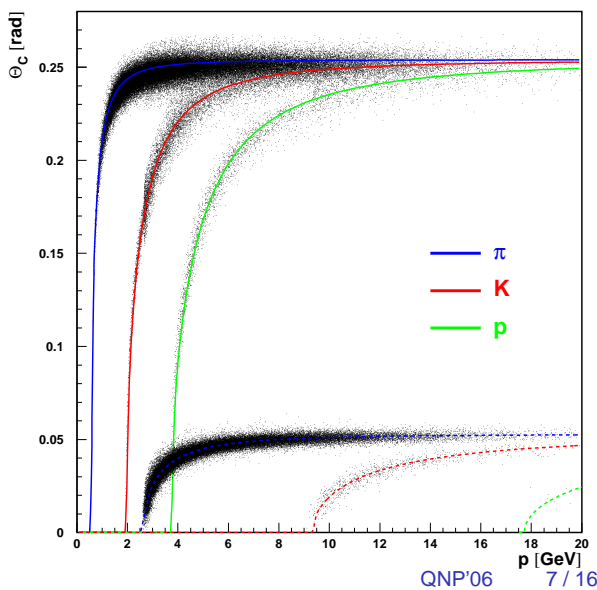




\section{The HERMES Spectrometer}

Hadron/lepton separation: combination of

- TRD

- Calorimeter

- Preshower

- $\mathrm{RICH}$

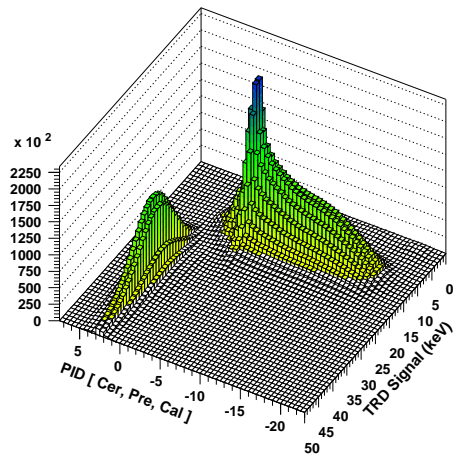

Hadron identification: dual radiator $\mathrm{RICH}$

- aerogel: $n=1.03$

- $\mathrm{C}_{4} \mathrm{~F}_{10}$ gas: $n=1.0014$

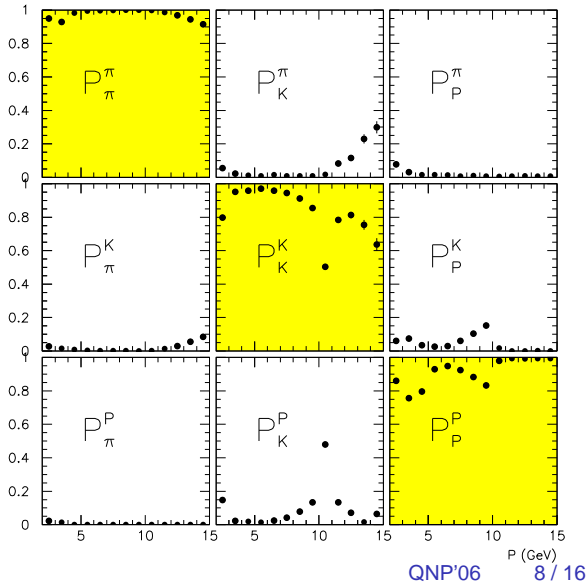




\section{Event selection and reconstruction}

$209.2 \mathrm{pb}^{-1}$ of deuterium data

- $e^{+} D \rightarrow \Lambda(1520) X \rightarrow p K^{-} X$

- $e^{+} D \rightarrow \bar{\Lambda}(1520) X \rightarrow \bar{p} K^{+} X$

Event topology (checked in Monte Carlo)

- $p$ and $K$ tracks closer than $0.6 \mathrm{~cm}$

- $\Lambda(1520)$ decayed inside target

- $R<0.4 \mathrm{~cm}$

- $|z|<18 \mathrm{~cm}$

- $\Lambda(1520)$ decay length $<5 \mathrm{~cm}$ (motivated by resolution) 


\section{Invariant mass spectra}

Acceptance correction (MC)

- Acceptance varies in $\Lambda(1520)$ mass region

- Shape of peak changes to skewed Breit-Wigner

- Mass from simple Breit-Wigner 1.5 $\pm 0.5 \mathrm{MeV}$ too high

Unbinned maximum likelihood fi t

- Breit-Wigner resonance shape

- Convolved with Gaussian detector resolution (4 MeV)

- Polynomial background (third order)

- Procedure:

- Determine the $M$ and $\Gamma$ from $\Lambda(1520) \rightarrow p K^{-}$spectrum

- Fix $M$ and $\Gamma$ for $\bar{\Lambda}(1520) \rightarrow \bar{p} K^{+}$spectrum 


\section{Invariant mass spectra}

\section{$\Lambda(1520) \rightarrow p K^{-}$}

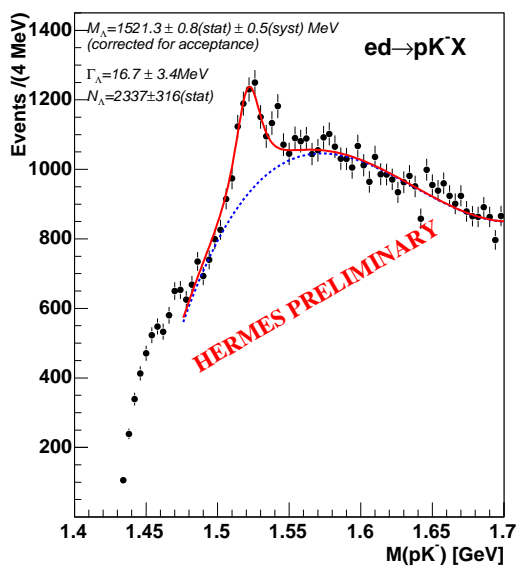

- $M=1521.3 \pm 0.8$ (stat) \pm 0.5 (syst) MeV

- $\Gamma=16.7 \pm 3.4 \mathrm{MeV}$
$\bar{\Lambda}(1520) \rightarrow \bar{p} K^{+}$

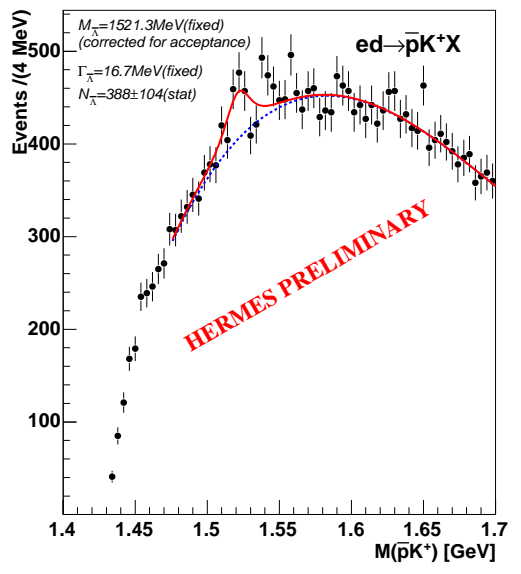

- $M_{P D G}=1519.5 \pm 1.0 \mathrm{MeV}$

- $\Gamma_{P D G}=15.6 \pm 1.0 \mathrm{MeV}$ 


\section{Partial cross sections and ratio}

Method of calculation (total cross section)

$$
\sigma_{\gamma^{*} D \rightarrow \Lambda(1520) X}=\frac{N_{\text {observed }}^{e D \rightarrow \Lambda(1520) X}}{\Phi \cdot B r \cdot \mathcal{L} \cdot \epsilon}
$$

- photon flux $\Phi=0.02 \mathrm{GeV}^{-3}$

- branching ratio $\mathrm{Br}=22.5 \%$

- integrated luminosity $\mathcal{L}=209.2 \mathrm{pb}^{-1}$

- efficiency and acceptance $\epsilon \ldots$ (full momentum range)

Effi ciency and acceptance $\epsilon$

- Determine $\epsilon$ using Monte Carlo simulation

- Pythia Monte Carlo: no $\Lambda(1520)$ state available...

- Model for decay momentum distribution of $\Lambda(1520)$ necessary 


\section{Partial cross sections and ratio}

Effi ciency and acceptance $\epsilon$

- Previous results $\left(\Theta, \Xi^{--}\right)$: assumed $\Xi^{0 *}$ momenta

- Now: looked at several hyperons $\left(\Lambda, \Sigma, \Xi, \Sigma^{*}, \Xi^{*}\right)$

- $\epsilon$ depends strongly on decay momentum distribution

- $0.03-3 \%$ for full momentum range, depending on model

- In HERMES acceptance $\left(P_{z}>6 \mathrm{GeV}\right)$ : momenta very similar

- $3-4 \%$ inside acceptance

- Differences from behavior outside HERMES acceptance

Polarization $\Lambda(1520)$

- $\Lambda(1520)$ has spin $J=\frac{3}{2} \rightarrow$ momentum distributions change

- Presented results assume isotropic decay $(J=0)$

- Effect of $J \neq 0$ not in systematic uncertainty 


\section{Partial cross sections and ratio}

$P_{z}>6 \mathrm{GeV}$

- Motivated by HERMES acceptance for $\Lambda(1520)$

Photo-production cross sections

- $\sigma_{\gamma^{*} D \rightarrow \Lambda(1520) X}=65.3 \pm 8.8$ (stat) \pm 6.9 (syst) $\mathrm{nb}$

- $\sigma_{\gamma^{*} D \rightarrow \bar{\Lambda}(1520) X}=9.8 \pm 2.6$ (stat) \pm 0.9 (syst) $\mathrm{nb}$

Cross section ratio $\bar{\Lambda}(1520)$ over $\Lambda(1520)$

- $R_{\bar{\Lambda} / \Lambda}=0.15 \pm 0.05$ (stat) \pm 0.02 (syst) 


\section{Extrapolation to $\bar{\Theta}(1540)$}

Assumptions

- Production mechanism $\Lambda(1520)$ and $\Theta(1540)$ similar

- Cross section ratio $\Lambda(1520)$ and $\Theta(1540)$ equal

Expected number of $\bar{\Theta}(1540)$

- $59 \pm 16 \Theta(1540)$ observed

- $10 \pm 4 \bar{\Theta}(1540)$ are expected when $R_{\bar{\Theta} / \Theta}=R_{\bar{\Lambda} / \Lambda}$

- $3 \pm 6 \bar{\Theta}(1540)$ were observed 


\section{Conclusions}

\section{Summary}

- Partial cross sections of the $\Lambda(1520)\left(P_{z}>6 \mathrm{GeV}\right)$ presented:

- $\sigma_{\gamma^{*} D \rightarrow \Lambda(1520) X}=65.3 \pm 8.8$ (stat) \pm 6.9 (syst) $\mathrm{nb}$

- $\sigma_{\gamma^{*} D \rightarrow \bar{\Lambda}(1520) X}=9.8 \pm 2.6$ (stat) \pm 0.9 (syst) $\mathrm{nb}$

- Ratio of $\bar{\Lambda}(1520)$ over $\Lambda(1520)$ production

- $R_{\bar{\Lambda} / \Lambda}=0.15 \pm 0.05$ (stat) \pm 0.02 (syst)

- Assuming similar $\Theta(1540)$ and $\Lambda(1520)$ production mechanisms, the expected number $\bar{\Theta}(1540)$ is $10 \pm 4$, and $3 \pm 6$ were observed.

Plans

- Data taking on hydrogen and deuterium is continuing

- Analysis of hydrogen data ongoing 